\title{
DESARROLLANDO COMPETENCIAS INVESTIGATIVAS EN LOS ESTUDIANTES DE EDUCACIÓN A TRAVÉS DEL APRENDIZAJE SITUADO
}

\section{DEVELOPING RESEARCH COMPETENCIES IN EDUCATION STUDENTS THROUGH SITUATED LEARNING}

\author{
Patricia Nakamura Goshima \\ Carol Rivero Panaqué \\ Alonso Velasco Tapia
}

\begin{abstract}
Resumen
El presente artículo presenta una experiencia docente realizada en una Facultad de Educación de Lima, Perú, en la que se planteó la vivencia de situaciones de aprendizaje situado. Los estudiantes realizaron una investigación exploratoria en una institución educativa pública ubicada en una zona urbano-marginal de la ciudad capital. La finalidad de la experiencia fue promover la adquisición de competencias investigativas en nuestros estudiantes a través de actividades conectadas con situaciones reales de las instituciones de educación básica. Como resultado, encontramos que los estudiantes reconocen el logro de competencias investigativas, como la capacidad para buscar información de distintas
\end{abstract}


bases de datos y fuentes confiables; la capacidad para plantear el método de investigación, y las técnicas e instrumentos para recoger la información; y la capacidad para aplicar los instrumentos de recolección de datos. Finalmente, los estudiantes pudieron responder a un problema de la vida real logrando un aprendizaje significativo.

\title{
Palabras Clave
}

Aprendizaje situado, competencias investigativas, educación superior.

\begin{abstract}
This article presents a teaching experience carried out in a faculty of Education in the city of Lima, in which the students experienced learning situations that consisted in carrying out exploratory research in teams, at a public educational institution located in a marginal urban zone of the capital city. The purpose of the experience was to promote the acquisition of investigative skills in our students through situated learning activities, connected with real situations in institutions of basic education. As a result, we found that students recognize the achievement of research competencies such as the ability to search for information from different databases and reliable bibliographic sources; the ability to plan the method, type of research, and the techniques and tools to collect the information; and the ability to apply the instruments to acquire the data. Also, the students were able to respond to a real-life problem and achieve meaningful learning.
\end{abstract}

\section{Key Words}

Situated learning, investigative competencies, higher education.

\section{Introducción}

En la sociedad actual, los estudiantes universitarios están expuestos a gran cantidad de información de manera permanente y a través de distintos medios. Ello hace necesario el desarrollo de competencias para ser capaces de seleccionar, organizar y realizar juicios 
racionales y críticos sobre la base de evidencias válidas, para así generar nuevos tipos de conocimiento y resolver los problemas a los que se enfrentarán en su vida profesional, proponiendo soluciones creativas.

Como futuros docentes, los estudiantes de Educación requieren especialmente de competencias investigativas que les permitan resolver las complejas situaciones que se presentan dentro de su quehacer diario, siendo generadores de conocimiento y produciendo investigación centrada en las cuestiones que surgen de su propia aula, de manera cotidiana, pero con una mirada crítica. A través de estas competencias, los estudiantes serán capaces de recoger la información de manera ordenada, documentando y analizando las experiencias dentro y fuera del aula, por lo que se requiere que desarrollen competencias investigativas que les permitan ser agentes activos en la mejora de los procesos educativos.

Para el logro de las competencias en la educación superior, diversas investigaciones demuestran la importancia del aprendizaje a través de situaciones que sean lo más reales posibles a las que el estudiante se enfrenta en su vida diaria (Anderson, Reder, \& Simon, 1996; Cunningham \& Dawes, 2016; González-Davies \& Enríquez-Raído, 2016; Lombardi, 2007; Stein, Isaacs, \& Andrews, 2004). Estas se desarrollan a través de actividades vivenciales, experienciales y formativas que permiten un aprendizaje significativo, y se sustenta en la idea de que aprender y hacer son acciones inseparables. Por ello, los estudiantes deben aprender en contextos pertinentes, desde una visión situada del aprendizaje que plantee un alto grado de relevancia social y cultural (F. Díaz Barriga, 2003; Lombardi, 2007).

Este artículo presenta la experiencia docente llevada a cabo en una Facultad de Educación de la ciudad de Lima, en la que se planteó la vivencia de situaciones de aprendizaje que permitieran a los estudiantes resolver problemas o necesidades reales que afrontan las instituciones de educación básica. Los estudiantes realizaron investigaciones grupales en instituciones educativas públicas ubicadas en zonas urbano-marginales de la ciudad de Lima.

La finalidad de la experiencia fue promover la adquisición de competencias investigativas en nuestros estudiantes a través de actividades de aprendizaje situado, conectadas con situaciones reales de las instituciones de educación básica. De este modo, se les comprometió en actividades en las que tuvieron que conjugar sus conocimientos conceptuales, procedimentales y actitudinales para poder resolver problemas reales de las instituciones.

\section{El desarrollo de las competencias y el aprendizaje situado a nivel universitario}

Aprender haciendo es generalmente considerada la manera más efectiva de aprender (Lombardi, 2007). Por otra parte, el aprendizaje situado es una modalidad que se origina en la práctica, en situaciones significativas de la vida real, lo cual exige la participación activa, dinámica y consciente por parte del estudiante. 
En este enfoque de aprendizaje, todo conocimiento se define como situado debido a que ocurre en un contexto determinado, como resultado de la actividad, el contexto, la cultura, y la interacción social con otras personas (F. Díaz Barriga, 2003). Por lo tanto, como sostienen Ferreyra y Fornasari (2016), los procesos de aprendizaje se desarrollan a través de actividades, situaciones y acontecimientos intersubjetivos que generan prácticas culturales, permitiendo la integración gradual de las personas a su comunidad, entorno y cultura. Se puede afirmar de esta manera, que el paradigma de aprendizaje situado va más allá de tan sólo "hacer" y realizar actividades, pues involucra una práctica social dentro de un contexto en el que el estudiante participa en una comunidad (Wenger, 2018).

Diversos estudios "hacen énfasis en que no tiene sentido ningún contenido escolar, si no es aprendido en un contexto de gran intensidad, la que proviene de la realidad" (A. Díaz Barriga, 2006, p. 18). Este autor afirma que para el logro de las competencias de nuestros estudiantes es necesario movilizar la información en los procesos educativos y luchar contra el enciclopedismo en la educación. Asimismo, señala que las competencias se logran cuando el estudiante consigue integrar la información a partir de problemas que provienen de la realidad, por lo que la perspectiva del aprendizaje situado se articula con claridad en esta posición.

Así, las actividades auténticas de aprendizaje presentan ciertas características esenciales, tales como: a) relevancia en el mundo real, con tareas funcionales, vinculadas a conflictos y problemas que resulten significativos para el estudiante; b) complejidad de la tarea, pues implica un reto, conllevando a una diversidad de resultados o formas de solucionarlos; c) posibilidad de ser examinadas desde distintas perspectivas utilizando variedad de recursos; d) fomento de la colaboración y la promoción de oportunidades para reflexionar, poniendo en práctica las creencias y valores (Lombardi, 2007; Monereo, SànchezBusqués, \& Suñé, 2012; Reeves, Herrington \& Oliver, 2002).

Asimismo, para el logro de un aprendizaje situado es necesario que este vaya de la mano con estrategias de enseñanza y aprendizaje que sean reflexivas, experienciales y que permitan transformar las prácticas cotidianas del aula (F. Díaz Barriga, 2006). Estas estrategias pueden ser: el análisis de casos, la solución de problemas auténticos, el método de proyectos, aprendizaje en el servicio, prácticas situadas o aprendizaje in situ en escenarios reales, simulaciones situadas, trabajo en equipos cooperativos y el aprendizaje mediado por las nuevas tecnologías, entre otras (F. Díaz Barriga, 2003). Para el desarrollo de la presente experiencia, se puso en práctica el aprendizaje in situ en escenarios reales, en los cuales los estudiantes, tal como se mencionó anteriormente, aplicaron sus conocimientos conceptuales, procedimentales y actitudinales para poder plantear alternativas de solución a los problemas reales de las instituciones. 


\section{El desarrollo de las competencias investigativas en Educación}

La necesidad de formar a los estudiantes universitarios en competencias investigativas se justifica principalmente por el contexto en el que vivimos actualmente, ya que nos encontramos expuestos a gran cantidad de información de manera permanente y a través de distintos medios. Como señala Pozo (2002 citado en Loo Corey, 2017), somos "auténticos informívoros" (p. 18), pues necesitamos de información en nuestra vida diaria para poder desenvolvernos y sobrevivir, lo cual hace que tengamos la necesidad de aprender continuamente y a un ritmo acelerado. Esta avalancha de información requiere la capacidad de seleccionar, organizar y dar sentido de manera crítica a dicha información, siendo capaces de realizar juicios racionales basados en evidencias válidas (Brew, 2010).

Las habilidades para la indagación crítica son necesarias en la sociedad compleja en la que vivimos, y nuestros estudiantes requieren desarrollarlas para ser capaces de responder a las demandas profesionales que enfrentarán en un contexto social de permanentes cambios. Los estudiantes universitarios deben estar preparados para resolver la gran variedad de problemas impredecibles que se presentan, proponiendo soluciones creativas, así como generando y adquiriendo nuevos tipos de conocimiento y de pensamiento (Brew, 2010). Es por ello que necesitan desarrollar competencias investigativas que les permitan ser gestores del conocimiento, construyendo y difundiendo dicho conocimiento, a partir del análisis y la reflexión crítica y profunda de la realidad, para dar respuesta a los múltiples problemas sociales.

Lo que se pretende no es la formación de investigadores, sino de profesionales que estén comprometidos con la búsqueda del conocimiento, siendo la investigación parte de su quehacer diario. Más aún en el campo educativo, y como futuros docentes, nuestros estudiantes deben desarrollar habilidades investigativas que les permitan construir la teoría de su práctica pedagógica y resolver las complejas situaciones que emanan del proceso educativo. Entendida de una manera tradicional, la investigación en Educación es un proceso realizado por un experto externo a la institución y sus resultados están dirigidos mayormente a una audiencia académica. En este proceso, el rol del docente de aula es el de implementar los cambios sugeridos a raíz de las investigaciones (en las cuales él o ella no participa); mas no se espera que sea él o ella quien presente los problemas, los cuestione o sugiera soluciones, sino que debe llevar a cabo un currículo o una planificación diseñada por personas ajenas a su aula (Dana \& Yendol-Hoppey, 2014).

Frente a este paradigma tradicional de investigación en el aula se encuentra aquel en el que el rol del docente de aula se presenta como un generador de conocimiento que produce investigación centrada en las cuestiones que surgen de su propia aula, y que participa en todo el proceso de diseño, recolección e interpretación de la información (Dana \& Yendol-Hoppey, 2014). Estas mismas autoras señalan que una de las ventajas de este tipo de investigación realizada por los docentes es que ellos toman las decisiones con respecto a qué se debe investigar y qué se debe hacer con la información encontrada, basándose en un análisis crítico y cuidadoso de lo que sucede en sus aulas, su escuela y su distrito, con la finalidad de mejorar la práctica educativa. 
Es en este paradigma en el que se posiciona la presente experiencia, pues se entiende el rol del docente de aula como un profesional que posee una mirada crítica de los procesos que se llevan a cabo en su aula, para seguir buscando formas de mejorarlos. Vergara (2016) considera a la investigación docente como un estudio sistemático e intencional de la práctica educativa. Es sistemática porque se recoge la información de manera ordenada, documentando y analizando las experiencias al interior y fuera del aula. Asimismo, es intencional porque se planifica y realiza deliberadamente con la finalidad de producir nuevos conocimientos en el campo de la Educación.

En las últimas décadas, la investigación docente ha ido tomando mayor relevancia, siendo considerada un elemento central para el desarrollo profesional docente de alta calidad, pues se considera que favorece la resolución de problemas $\mathrm{y}$, al fortalecer la capacidad reflexiva de los docentes, es un ingrediente esencial para el logro de la mejora de su práctica (Ermeling, 2010). Las investigaciones demuestran que las escuelas necesitan ser un lugar de aprendizaje no solamente para los estudiantes, sino también para los maestros, como una condición necesaria para la mejora de la enseñanza en el aula y el logro de los estudiantes (Saunders, Goldenberg, \& Gallimore, 2009). La Facultad de Educación en la que se realizó esta experiencia no es ajena a esta tendencia, pues sigue un enfoque curricular que, entre otras características, busca formar a sus estudiantes en habilidades de investigación.

Al hablar de competencias en investigación en el campo educativo nos referimos a los conocimientos, destrezas y actitudes que posee la persona para aplicar métodos y técnicas de investigación con la finalidad de interpretar, argumentar y proponer alternativas frente a los problemas de la práctica docente, para con esto mejorar los procesos educativos (Muñoz Giraldo, Quintero Corzo, \& Munévar Molina, 2005). Así, en la presente experiencia se buscó desarrollar en los estudiantes de Educación las siguientes siete competencias investigativas:

Competencias

Dominio de la

literatura científica

Manejo de la
tecnología

Planificación

Diseño de la

investigación

Recolección de información

\section{Descripción}

Capacidad para buscar información de distintas bases de datos y fuentes bibliográficas confiables y desarrollar el marco conceptual.

Capacidad para utilizar herramientas tecnológicas (Ej: computadora, software especializado, procesador de datos, internet, etc.) para el desarrollo de la investigación.

Capacidad para hallar y definir un problema de investigación relacionado al campo educativo formulando un objetivo de investigación coherente (que responda a dicho problema). Capacidad para planear, organizar y ejecutar las actividades de investigación en función de un cronograma. Capacidad para plantear el método, tipo de investigación, y las técnicas e instrumentos para recoger la información. Capacidad para aplicar los instrumentos que permitan la adquisición de datos. 
Análisis de datos

Capacidad para organizar, procesar e interpretar datos cuantitativos y/o cualitativos, formulando los resultados de investigación.

Tabla 1. Competencias investigativas en Educación: Fuente: Elaboración propia.

A continuación, desarrollaremos los objetivos y la metodología aplicada en la presente experiencia docente.

\section{Objetivos}

- Promover la adquisición de competencias investigativas en los estudiantes a través de actividades conectadas con situaciones reales de las instituciones de educación básica.

- Desarrollar prácticas pedagógicas que trasciendan el aula y beneficien a la comunidad generando una constante reflexión en los estudiantes de la carrera de Educación sobre su propia práctica docente.

\section{Metodología}

El trabajo describe y evalúa una experiencia de enseñanza-aprendizaje desarrollada para la asignatura "Investigación y práctica educativa 4: La institución educativa" perteneciente al cuarto ciclo de estudios en Educación de una universidad privada de Lima. En particular, la asignatura se impartió como curso general de las Especialidades de Educación Inicial y Primaria durante el ciclo académico 2016-2 (agosto - diciembre).

Este curso, de tres créditos, forma parte del Plan de Estudios de diez ciclos de la Facultad de Educación (2016), y se espera que los estudiantes a través de esta asignatura logren la competencia de investigar permanentemente sobre los diferentes actores, componentes y procesos educativos en diversos escenarios sociales, para diseñar, desarrollar, sistematizar y validar propuestas de mejora que amplíen el conocimiento de manera crítica y reflexiva. En ese sentido, el curso tuvo como finalidad que los estudiantes conocieran las características y organización de una institución escolar a través del diseño y desarrollo de un diagnóstico institucional. Para ello, se les enseñó a manejar diferentes técnicas e instrumentos de recogida de información, como la observación, el análisis documental y la encuesta.

La metodología que se desarrolló en la asignatura fue activa y participativa, bajo una perspectiva de enseñanza-aprendizaje reflexiva y crítica en la que se buscó motivar, estimular y orientar el aprendizaje. Para ello, se realizaron distintas actividades evaluadas y de proceso de manera permanente, como analizar e interpretar material bibliográfico, exposiciones, diálogo y discusión de los principales contenidos del curso, elaboración de trabajos colaborativos e individuales de investigación y la aplicación de una investigación diagnóstica en una institución educativa localizada en una zona urbano-marginal. 
En el siguiente apartado describiremos con mayor detalle la propuesta de innovación considerando los participantes de este curso y las fases realizadas.

\section{Participantes:}

En esta asignatura se inscribieron un total de 80 alumnos ( 72 mujeres y 8 hombres) con edades que oscilaron entre los 18 y 27 años y de distintas realidades socioeconómicas, de los cuales 75 realizaron todas las actividades y 73 Ilenaron una encuesta sobre las competencias investigativas desarrolladas al finalizar el curso. Asimismo, estos estudiantes fueron divididos en tres grupos con sus respectivos docentes (dos mujeres y un hombre) entre los 38 y 40 años con dominio en investigación y experiencia de más de 10 años en la carrera de Educación.

\section{Procedimiento:}

Para la realización de esta innovación se consideraron tres fases (Figura 1). La primera consistió en la planificación de las estrategias y actividades del curso donde se identificaron las instituciones educativas públicas ${ }^{1}$ en las que se realizarían las visitas por parte de los estudiantes. Para ello, los docentes del curso visitaron las instituciones públicas urbanomarginales con la finalidad de hacer un primer reconocimiento del lugar y recoger información general. Luego, los estudiantes formados en equipos (cuatro por escuela) pudieron hacer una primera visita y con ello se hizo la formulación de la situación problemática de la entidad educativa y los objetivos del diagnóstico sobre la base de la información recogida. Además, con la finalidad de tener una mirada más amplia de la situación actual de la institución educativa, cada uno de los grupos de estudiantes consideró uno de los siguientes temas:

- Oferta y uso de servicios educativos: infraestructura (calidad de los materiales de pisos, paredes y techos); acceso a servicios básicos (agua potable, desagüe y energía eléctrica); equipamiento (disponibilidad de mobiliario y de equipos adicionales como bibliotecas, laboratorios, auditorios, etc.); y uso de las Tecnologías de la Información y Comunicación (TIC).

- Proceso de enseñanza: calendario escolar, tiempo de clases, uso del tiempo en actividades académicas, la formación inicial, continua y experiencia de los docentes.

\footnotetext{
1 Se seleccionó este tipo de instituciones con la finalidad de reconocer su realidad y los problemas más urgentes, así como los factores que están interactuando, de manera que este diagnóstico sea un punto de partida para que las instituciones puedan realizar mejoras en los diferentes ámbitos académicos y administrativos.
} 
- Clima institucional: capacitaciones, trabajo en equipo, nivel de satisfacción, comunicación, relaciones interpersonales, etc.

- Aspectos familiares de los estudiantes: lengua materna, nutrición, estructura familiar, acceso a servicios básicos y actividad económica que sostiene al hogar.

A continuación, se elaboró el marco conceptual (donde se hizo uso de fuentes diversas y confiables) y el marco contextual (donde se caracterizó a los sujetos que formaban parte de la institución y la realidad socioeducativa). Así también, se seleccionaron las técnicas (ya mencionadas anteriormente) y se elaboraron los instrumentos para la recogida de información.

En la segunda fase se aplicaron los instrumentos en las escuelas con la finalidad de recoger la información y luego se hizo el análisis de datos a través de la selección, ordenamiento y procesamiento de información, tabulación y elaboración de gráficos. Con estos resultados se pudo hacer el diagnóstico en cada una de las instituciones educativas y la elaboración de las conclusiones y recomendaciones.

Finalmente, como tercera fase se hizo una sistematización de toda la experiencia en el curso dictado para evaluar el trabajo realizado y asegurar su continuidad y mejora. De esta manera, se realizó una encuesta a los estudiantes con la finalidad de saber las competencias investigativas que lograron a través de este curso.

\section{Fase 1}

Fase 2
Fase 3
- Actividad 1: Planificación de las estrategias y actividades del curso.

- Actividad 2: Visita de los docentes a las instituciones públicas para un primer reconocimiento del lugar $y$ la recogida de información general.

- Actividad 3: Formulación de la situación problemática y los objetivos del diagnóstico junto con los estudiantes, en base a la información recogida de las escuelas.

- Actividad 4: Elaboración del marco conceptual y contextual para el diagnóstico.

- Actividad 5: Selección de técnicas y elaboración de instrumentos para la recogida de información.
- Actividad 1: Aplicación de los instrumentos en las escuelas para la recogida de la información.

- Actividad 2: Selección, ordenamiento $y$ procesamiento de datos, tabulación, y elaboración de gráficos.

- Actividad 3: Descripción y análisis de la información.

- Actividad 4: Elaboración de conclusiones recomendaciones.
- Actividad 1: Revisión de los informes diagnósticos (por parte de los docentes del curso) $y$ sistematización de toda la experiencia para asegurar la continuidad y mejora de la misma.

- Actividad 2: Publicación de los resultados. 
Figura 1. Fases de la innovación docente. Fuente: Elaboración propia.

\section{Resultados}

Una vez obtenidas las respuestas se analizaron los datos, y los resultados los podremos observar a continuación.

\subsection{Dominio de la literatura científica}

La competencia seleccionada en primer lugar por los estudiantes como la que más desarrollaron durante la elaboración de su investigación fue la de "dominio de la literatura científica", que implica la capacidad para buscar información de distintas bases de datos y fuentes bibliográficas confiables, principalmente para la redacción y fundamentación del marco conceptual. Es decir, aproximadamente el $56 \%$ de los estudiantes priorizaron esta competencia y esto se debe a que, en la presente experiencia, de las 16 sesiones de clase que formaron parte del semestre, cuatro estuvieron destinadas a la búsqueda de información y a la elaboración del marco conceptual.

En estas sesiones de clase, los estudiantes formados en grupos desarrollaron actividades, como identificar y discriminar fuentes bibliográficas confiables, buscar artículos e investigaciones en diferentes bases de datos nacionales e internacionales, redactar de manera adecuada y coherente el marco conceptual y utilizar el formato APA (sexta edición). Dentro de los comentarios recogidos de los estudiantes, varios de ellos valoran la asesoría del docente del curso en dichos momentos, revisando junto con ellos la calidad de las fuentes y la redacción de su marco conceptual.

Así también, 27 estudiantes (38\%) consideraron que el desarrollo del marco conceptual fue lo más exigente de elaborar realizando algunos comentarios como:

"Lo más exigente fue el marco conceptual y el análisis debido a que se requería la búsqueda de autores y mucha fundamentación para realizar el informe" ( $\left.n^{\circ} 9\right)$.

"El marco conceptual, ya que necesitabas los datos precisos que serían fundamento para todo tu trabajo de investigación" ( $\left.n^{\circ} 25\right)$.

"En cuanto a redacción, la parte más exigente resultó ser el marco conceptual, pues implicaba una redacción coherente con todos los otros párrafos junto a una revisión detallada" ( $\left.n^{\circ} 27\right)$.

Como podemos ver en las respuestas, los estudiantes mencionan la importancia de fundamentar y redactar adecuadamente su marco conceptual y es a través de las actividades desarrolladas en las sesiones de clase del curso que han podido lograr este aprendizaje.

\subsection{Diseño de la investigación}


La competencia seleccionada en segundo lugar por los estudiantes fue la de "diseño de la investigación", que implica la capacidad de plantear el método, así como precisar el tipo de investigación y las técnicas e instrumentos que permitan recabar la información propuesta en la problemática inicial.

Aproximadamente el $45 \%$ de los estudiantes priorizaron esta competencia dentro de las más logradas en el curso, explicando que para ellos había sido la primera vez que planificaban una investigación. Además, para el planteamiento del diseño, durante tres sesiones los estudiantes elaboraron los instrumentos y posteriormente llevaron a cabo la recogida y análisis de la información.

La priorización de esta competencia como una de las más logradas dentro del curso responde a la exigencia que se le dio a la explicitación del método y de las categorías de análisis, como elementos esenciales para recolectar información que permita responder al objetivo de la investigación y al problema planteado inicialmente. Asimismo, una vez determinadas las categorías, cada equipo de estudiantes elaboró los instrumentos pertinentes, tales como: guía de entrevista, cuestionario y guía de observación.

Una vez que cada grupo elaboró sus instrumentos, se realizó una revisión por pares, con la finalidad de validarlos y verificar la coherencia y pertinencia con respecto al objetivo y el problema de investigación; así como la suficiencia para recoger la información sobre las categorías planteadas. En este proceso, cada equipo colaboró en la revisión de los instrumentos de los otros tres equipos del aula.

Como vemos, el proceso de diseño de la investigación buscó que los estudiantes participaran de manera activa y colaborativa, siendo capaces de entender la importancia de esta competencia en un diagnóstico educativo, ya que nos permite delimitar la forma en que se realizará la investigación, así como ordenar, esquematizar y registrar los datos.

\subsection{Recolección de información}

Ahora bien, aproximadamente un $42 \%$ de estudiantes reconocen que la competencia de recolección de información, que implica la capacidad para aplicar los instrumentos diseñados, también ha sido importante para el proceso de su investigación.

En el curso, la recolección de información se realizó luego de hacer una validación de los instrumentos y ajustes realizados por cada equipo de trabajo. Cabe precisar que la aplicación de los instrumentos se realizó durante la octava y novena semana del semestre y donde todos los estudiantes realizaron visitas a las instituciones educativas con la finalidad de recabar los datos necesarios para su investigación.

Igualmente, esta competencia fue mencionada por los estudiantes como una de las más difíciles, ya que precisaron que la recolección de información les demandaba mayor tiempo y dedicación. Esto se refleja en los siguientes comentarios: 
"La etapa más exigente de la investigación fue la recolección de los datos, sobre todo la transcripción y tabulación" ( $\left.n^{\circ} 49\right)$.

"El aplicar nuestros instrumentos, pues cuando al principio nos acercamos a los padres de familia, se negaban" ( $\left.n^{\circ} 20\right)$.

Como podemos ver en las respuestas, los estudiantes hacen mención a esta competencia como aquella que les demandó mayor tiempo y fue la que más trabajo generó, porque muchas veces la población seleccionada no brindaba el espacio para que los estudiantes entrevisten o puedan ser encuestados. Sin embargo, a pesar de ello, los estudiantes señalan que pudieron superar las dificultades y recoger la información que necesitaban para su investigación. De esta manera, el vencer los obstáculos les permitió sentir que desarrollaron esta competencia tan necesaria en el proceso de toda investigación.

\subsection{El aprendizaje situado como un aprendizaje "real"}

En la encuesta aplicada a los estudiantes también se les preguntó sobre la experiencia vivida para conocer si ellos consideraban que el curso, a través de las visitas de campo y todo el proceso realizado, les había brindado oportunidades reales de aprendizaje, así como el motivo de su respuesta. Frente a esta pregunta, del total de 73 estudiantes, la gran mayoría (70) respondió que el curso sí les había brindado oportunidades reales de aprendizaje, 2 respondieron que les brindó muy pocas oportunidades reales de aprendizaje y 1 persona no respondió la pregunta.

Al ser una pregunta abierta, las respuestas fueron muy diversas, pero se encontró que la mayor parte de los estudiantes hicieron referencia a la experiencia y el contacto directo con la realidad como un elemento esencial para el logro de sus aprendizajes, tal como lo señala un estudiante:

"A través de las salidas de campo en forma constante se pudo contrastar la teoría con la realidad de las instituciones educativas, mediante el ejercicio de la observación, las entrevistas y encuestas" ( $\left.n^{\circ} 26\right)$.

Del total de respuestas, el término que más se repite en ellas es el de "realidad" o "realidades", haciendo alusión al conocimiento que lograron alcanzar sobre la realidad o las distintas realidades de las instituciones educativas visitadas. Encontramos este término en forma singular o plural en 23 de las 73 encuestas de los estudiantes. Si tomamos en cuenta términos similares, como el conocimiento de distintas "situaciones" y "contextos", son 27 las respuestas en las que los encontramos. De este modo, vemos que más de un tercio de los estudiantes $(37 \%)$ reconocen que lograron un mayor conocimiento de la realidad a través de las experiencias vividas en el curso.

En segundo lugar, el término más utilizado por los estudiantes fue "observar" u "observación", el cual identificamos en 10 respuestas de los estudiantes. Esta respuesta es 
significativa porque hace énfasis en el contacto directo por parte de los estudiantes con el fenómeno educativo sobre el cual recogieron información para realizar sus investigaciones.

"Considero que he podido aprender mucho gracias a la observación. La observación enfocada en el tema de investigación me ha permitido ser consciente de más cosas" ( $n^{\circ}$ 27).

Finalmente, el término "práctica" tiene 5 coincidencias, en las cuales los estudiantes resaltan la importancia de la acción y participación directa en el proceso de aprendizaje.

"Sí, absolutamente, porque hemos podido contrastar la teoría con la práctica. Es la primera vez que realizo un diagnóstico con un grupo y siento que me ha ayudado en mi formación, no solo personal sino también intelectual" ( $\left.n^{\circ} 44\right)$.

Dentro de esta diversidad de respuestas, los estudiantes señalan la importancia del aprendizaje a través de la participación activa, la experimentación, la vivencia y el contacto directo con la realidad como elementos esenciales para su aprendizaje. Podemos afirmar que casi la totalidad de los estudiantes reconocen que lograron aprender a través de estas situaciones reales en las que ellos llevaron a la práctica sus conocimientos y habilidades. De esta forma, fueron capaces de construir su propio aprendizaje desde las experiencias a las que se enfrentaron, en un contexto de interacción social.

\section{Consideraciones finales}

Como podemos ver, en esta experiencia docente se vivenciaron situaciones de aprendizaje situado a través del diagnóstico desarrollado en las diferentes instituciones educativas públicas, promoviendo así las competencias investigativas en los estudiantes de una facultad de Educación de Lima. Es decir, las estrategias metodológicas activas que consideran situaciones auténticas de aprendizaje ayudan a lograr las competencias investigativas en los estudiantes despertando su interés y motivación, y fortaleciendo el logro de un aprendizaje significativo y duradero.

Entre las competencias que los estudiantes lograron desarrollar se encuentran: el dominio de la literatura científica mediante la búsqueda de la información y la elaboración del marco conceptual; el diseño de la investigación, en donde se desarrolló el método, tipo de investigación y las técnicas para recoger la información; y como tercera competencia, la recolección de información mediante la aplicación de los instrumentos que permitieron obtener datos para el estudio.

Otro punto importante que consideró la mayoría de los participantes fue que el curso permitió un aprendizaje mediante la experiencia y el contacto directo con la realidad contrastando de esta manera la teoría con la práctica y ayudando así a su formación personal y profesional. Por lo tanto, el desarrollo de prácticas pedagógicas que trascienden el aula fortalece el compromiso de los estudiantes con la comunidad, generando una reflexión sobre su práctica docente, comprendiendo la realidad de la educación nacional y actuando en beneficio de ella. 
Adicionalmente, a través de esta experiencia rescatamos como lecciones aprendidas dentro del curso:

- La importancia de la coordinación y comunicación entre los docentes del curso, así como el apoyo logístico y administrativo por parte de la Facultad para el proceso de investigación en las diferentes instituciones educativas.

- La necesidad de una planificación y elaboración de materiales didácticos antes del inicio del semestre para prever las actividades que se deben desarrollar durante el curso.

- La necesidad del acompañamiento a los estudiantes por parte de los docentes del curso en cada visita realizada a las instituciones educativas, con la finalidad de resolver las situaciones imprevistas que se presenten.

\section{Bibliografía}

Anderson, J. R., Reder, L. M., \& Simon, H. A. (1996). Situated learning and education. Educational Researcher, 25(4), 5-11. (DOI: https://doi.org/10.3102/0013189X025004005)

Brew, A. (2010). Imperatives and challenges in integrating teaching and research. Higher Education Research \& Development, 29(2), 139-150. (DOI: 10.1080/07294360903552451)

Cunningham, I., \& Dawes, G. (2016). The handbook of work based learning. London: Routledge.

Dana, N. F., \& Yendol-Hoppey, D. (2014). The reflective educator's guide to classroom research: Learning to teach and teaching to learn through practitioner inquiry. Thousand Oaks: Corwin Press.

Díaz Barriga, A. (2006). El enfoque de competencias en la educación: ¿Una alternativa o un disfraz de cambio? Perfiles educativos, 28(111), 7-36.

Díaz Barriga, F. (2003). Cognición situada y estrategias para el aprendizaje significativo. Revista electrónica de investigación educativa, 5(2), 1-13. Recuperado de https://redie.uabc.mx/redie/article/view/85

Díaz Barriga, F. (2006). Enseñanza situada: vínculo entre la escuela y la vida. Ciudad de Mexico: McGraw-Hill.

Ermeling, B. A. (2010). Tracing the effects of teacher inquiry on classroom practice. Teaching and teacher education, 26(3), 377-388. (DOI: 10.1016/j.tate.2009.02.019) 
Facultad de Educación (2016). Plan de estudio. Recuperado de http://files.pucp.edu.pe/estudiante/2016/08/09152644/plan_de_estudios_educacion_2016 $-2 . p d f$

Ferreyra, Y. M., \& Fornasari, M. L. (2016). Aprendizaje significativo en prácticas preprofesionales: Una experiencia-acontecimiento en la formación de psicólogos educacionales. Educación, Formación e Investigación., 2(4). Recuperado de http://ppct.caicyt.gov.ar/index.php/efi/article/view/8563/9077

González-Davies, M., \& Enríquez-Raído, V. (2016). Situated learning in translator and interpreter training: bridging research and good practice, The Interpreter and Translator Trainer, 10(1), 1-11. (DOI: 10.1080/1750399X.2016.1154339)

Lombardi, M. M. (2007). Authentic learning for the $21^{\text {st }}$ century: An overview. Educause learning initiative, 1, 1-12. Recuperado de http://www.Imi.ub.edu/cursos/s21/REPOSITORIO/documents/Lombardi_2007_Authentic_ learning.pdf

Loo Corey, C. (2017). Enseñar a aprender: Desarrollo de capacidades-destrezas en el aula. Santiago de Chile: Arrayán Editores.

Monereo, C., Sànchez-Busqués, S., \& Suñé, N. (2012). La enseñanza auténtica de competencias profesionales. Un proyecto de aprendizaje recíproco institutouniversidad. Profesorado. Revista de Currículum y Formación de Profesorado, 16(1), 79101. Recuperado de http://www.ugr.es/ recfpro/rev161ART6.pdf

Muñoz Giraldo, J. F., Quintero Corzo, J., \& Munévar Molina, R. A. (2005). Competencias investigativas para profesionales que forman y enseñan: ¿cómo desarrollarlas? Bogotá: Cooperativa Editorial Magisterio.

Reeves, T. C., Herrington, J., \& Oliver, R. (2002). Authentic activities and online learning. Hersda, 562-567. Recuperado de https://www.researchgate.net/publication/49283243_Authentic_activities_and_online_lear ning

Saunders, W. M., Goldenberg, C. N., \& Gallimore, R. (2009). Increasing achievement by focusing grade-level teams on improving classroom learning: A prospective, quasiexperimental study of Title I schools. American Educational Research Journal, 46(4), 1006-1033. (DOI: 10.3102/0002831209333185)

Stein, S. J., Isaacs, G., \& Andrews, T. (2004). Incorporating authentic learning experiences within a university course. Studies in Higher Education, 29(2), 239-258. (DOI: 10.1080/0307507042000190813) 
Vergara, M. (2016). La práctica docente. Un estudio desde los significados. Revista Cumbres, 2(1), 73-99. Recuperado de https://dialnet.unirioja.es/descarga/articulo/6550779.pdf

Wenger, E. (2018). A social theory of learning. En K. Illeris (Ed.), Contemporary Theories of Learning: learning theorists... in their own words. Oxford: Routledge.

\section{REFERENCIA BIBLIOGRÁFICA}

Nakamura Goshima, P.; Rivero Panaqué, C. y Velasco Tapia, A. (2018). Desarrollando competencias investigativas en los estudiantes de Educación a través del aprendizaje situado. Aula de Encuentro, 21(1), pp. 182-197.

Patricia Nakamura Goshima es Profesora Auxiliar del Dpto. Académico de Educación de la Pontificia Universidad Católica del Perú

Correo-e: pnakamura@pucp.pe

Carol Rivero Panaqué es Profesora Asociada del Dpto. Académico de Educación de la Pontificia Universidad Católica del Perú Correo-e: crivero@pucp.pe

Alonso Velasco Tapia es Profesor Contratado del Dpto. Académico de Educación de la Pontificia Universidad Católica del Perú Correo-e: velasco.ag@pucp.edu.pe 